Revista Destaques Acadêmicos, Lajeado, v. 10, n. 3, 2018. ISSN 2176-3070

DOI: http://dx.doi.org/10.22410/issn.2176-3070.v10i3a2018.1981

http://www.univates.br/revistas

\title{
MAPEAMENTO DE FOCOS DE CALOR NO MUNICÍPIO DE JUARA/MT NOS ANOS DE 2016 E 2017: CONSTRUÇÃO DE BANCO DE DADOS PARA ANÁLISES AMBIENTAIS REGIONAIS
}

\author{
Luciano Aparecido de Oliveira ${ }^{1}$, André Jasper ${ }^{2}$
}

\begin{abstract}
Resumo: $\mathrm{O}$ fogo é constantemente apontado como uma ameaça à preservação ambiental devido ao seu poder de degradação. Entretanto, ao longo das últimas décadas, pontos positivos da ocorrência de incêndios vegetacionais têm sido apresentados para alguns ecossistemas ao redor do mundo. No contexto de evolução de biomas terrestres ao longo do tempo, o fogo pode ser de grande importância quando associado a ecossistemas em que se faz necessário para o desenvolvimento de diferentes espécies de plantas. Para que seja possível compreender o papel desse tipo de evento nos sistemas em que pode ser considerado "natural" é necessário, no primeiro momento, definir a sua origem (natural ou antrópica) e identificar em qual proporção determinadas regiões são acometidas pela sua manifestação. Essa pesquisa investigou a ocorrência de incêndios vegetacionais no Município de Juara, Região Noroeste do Estado de Mato Grosso, no Bioma Amazônia e na Amazônia Legal no período de 2016 a 2017 (02 anos). Com base em análises de Sensoriamento Remoto através do Banco de Dados da Plataforma do INPE Queimadas foi possível quantificar o número de focos calor de cada ano e identificar os meses de maior incidência. Em seguida comparamos os resultados com pesquisas também realizadas na Amazônia Legal.
\end{abstract}

Palavras-chave: Uso Antrópico do Fogo. Amazônia Legal. Ecologia do Fogo. Manejo do Fogo. Incêndios Vegetacionais.

1 Graduado em Administração pela Universidade Federal de Mato Grosso, Professor no Curso de Adminstração da Universidade Estadual do Mato Grosso e Mestrando do Programa de Pós-Graduação em Ambiente e Desenvolvimento da Univates. lucianoolivetto@hotmail.com

2 Biólogo, Mestre e Doutor em Geociências pela Universidade Federal do Rio Grande do Sul, Pós Doutor. Professor do Programa de Pós-Graduação em Ambiente e Desenvolvimento. A.jasper@univates.br 


\section{INTRODUÇÃO}

Nas últimas décadas os estudos sobre o fogo tiveram vários focos de pesquisa interdisciplinar com a proposta de compreender sua função biológica, ecológica e histórica (SCOTT et al., 2014). Estudos exemplificam a importância do ciclo do fogo na evolução (AVITABILE et al., 2013) e sua participação onipresente nos distúrbios dos ecossistemas (LARA et al., 2017).

É possível identificar diversas espécies que criam e mantém seus habitats em função do fogo, entretanto, a interferência do homem altera sua intensidade, causando ameaças a algumas espécies (AVITABILE et al., 2013).

Burrows (2008) aponta que o fogo controlado, quando sistematizado e utilizado de forma proativa, além de diminuir a incidência de incêndios vegetacionais incontroláveis, causadores de diversos prejuízos de ordem econômica e social, contribui para a conservação da biodiversidade. O autor utiliza como base para a sua afirmação o fato de que alguns sistemas florestais necessitam de fogo e, consequentemente, de uma variação na sua "estabilidade ecológica" para possibilitar o desenvolvimento de seu potencial de biodiversidade. Dessa forma, os efeitos negativos de um incêndio vegetacional seriam reduzidos.

O fogo é utilizado de diferentes maneiras em cada região do mundo. Chen et al. (2017) descreve que no sudoeste da China, os incêndios ocorrem regularmente em terras agrícolas. Pyne (2012) descreve que na Suécia e na Austrália, os incêndios florestais acontecem devido ao manejo do campo na prática da agricultura, onde o fogo é usado como método de prevenção de grandes incêndios e para regeneração da área.

No Brasil, Alvarado et al. (2017) contextualiza que o uso do fogo molda a composição das Savanas de Cerrado, seja pela ação antrópica ou natural, principalmente pelo Cerrado ter combustíveis secos, devido às condições climáticas e propícias para incidência de altas temperaturas.

Para Scott et al. (2014) no contexto histórico evolutivo dos ecossistemas e do homem, o fogo tem exercido um importante papel, principalmente no manejo de biomas, que se faz elemento natural e necessário. $\mathrm{O}$ autor afirma que a compreensão fogo só é possível com a prática de pesquisas que visam compreender seu papel biológico, ecológico e histórico, para trazer a luz da ciência seu impacto ao meio ambiente.

Scott et al. (2014) descreve que existiam poucos estudos sobre o fogo, mas que nos últimos 20 anos as pesquisas e conferências sobre o tema aumentaram. Para o autor cada pesquisador deve abordá-lo de acordo com sua área de interesse e formação.

Avitabile et al. (2013) ressalta que as pesquisas sobre o fogo são importantes em um panorama multidisciplinar, pois contribuem para a ecologia, para a química, para o manejo e também para o controle de queimadas. 
Possibilitando informações e conhecimentos que auxiliam na tomada de decisão sobre o uso do fogo.

Compreender o fogo e sua interação com o ambiente através da ecologia permite conhecer o papel natural desse elemento, que ao longo da história exerceu papel fundamental na evolução do homem. Enquanto administrador possibilita confrontar seu papel natural à realidade ambiental promovida pelo desenvolvimento econômico, através do Agronegócio. Cenário qual o fogo é utilizado como modelador de ambientes, em prol da produção e do capital com interface a segurança alimentar.

A geração de energia, a produção de alimentos e fibras está conectada a problemas contemporâneos na garantia da segurança alimentar, tais como o aquecimento global, poluição, queimadas e problemas persistentes ao acesso da população aos alimentos (ZYLBERSZTAJN, 2017).

Dados do Instituto Brasileiro de Geografia e Estatística (IBGE; 2016) apontam que em 2016 no Brasil foram identificados através dos satélites NOAA e AQUA UMD o total de 188.467 focos de calor. No mesmo ano o Estado de Mato Grosso foi destaque na incidência de queimadas, com 29.571 focos de calor identificados, normalmente relacionados a práticas de manejo nos campos para produção de agricultura e bovinocultura.

Nesse ambiente destaca-se o Município de Juara (localizado na região noroeste do estado de Mato Grosso) que possui economia baseada no agronegócio e que segundo Coutinho (2004) apresenta altos índices de queimadas.

Essa pesquisa teve como objetivo identificar a incidência de focos de calor no município de Juara- Mato Grosso nos anos de 2016 e 2017 utilizando o banco de dados do Instituto Nacional de Pesquisas Espaciais. O mapa e as tabelas elaborados ajudam a compreender a dinâmica do fogo e podem ser utilizados para comparações e correlações com variáveis ambientais.

\section{MATERIAS E MÉTODOS}

\section{1 ÁREA DE ESTUDO}

O estado do Mato Grosso (FIGURA 1; B), com 903.357,91 km², está localizado na região Centro-Oeste do Brasil, e em seu território ocorrem três dos biomas brasileiros (Amazônia, Cerrado e Pantanal) estabelecidos pelo IBGE (IBGE, 2004). O município de Juara (FIGURA 1; C), objeto do presente estudo, encontra-se na porção noroeste do estado e está inserido no Bioma Amazônia e na Amazônia Legal (IMEA, 2017; IBGE, 2004) (FIGURA 1).

O processo de colonização e ocupação da região foi intensificado a partir da década de 1970 com políticas públicas de ocupação de "espaços vazios", promovidas pelo Governo Brasileiro (MORENO, 2005). Esta iniciativa visava 
a conquista e exploração de novas área de produção agropastoril, sendo que o município de Juara foi reconhecido como unidade administrativa em dezembro de 1981 (IBGE, 2016).

Atualmente a população do município de Juara é estimada em 32.791 habitantes, sendo 26.031 habitantes no centro urbano e 6.738 habitantes em propriedades rurais (IBGE, 2017). A economia local é baseada na agricultura, comércio e agropecuária, com destaque a produção de gado de pasto com rebanho de 945.201 mil cabeças (IMEA, 2017), o terceiro maior rebanho do estado de Mato Grosso.

Figura 1 - Mapa de localização geográfica da área de estudo, com destaque à malha hidrográfica do município de Juara, Mato Grosso, Brasil

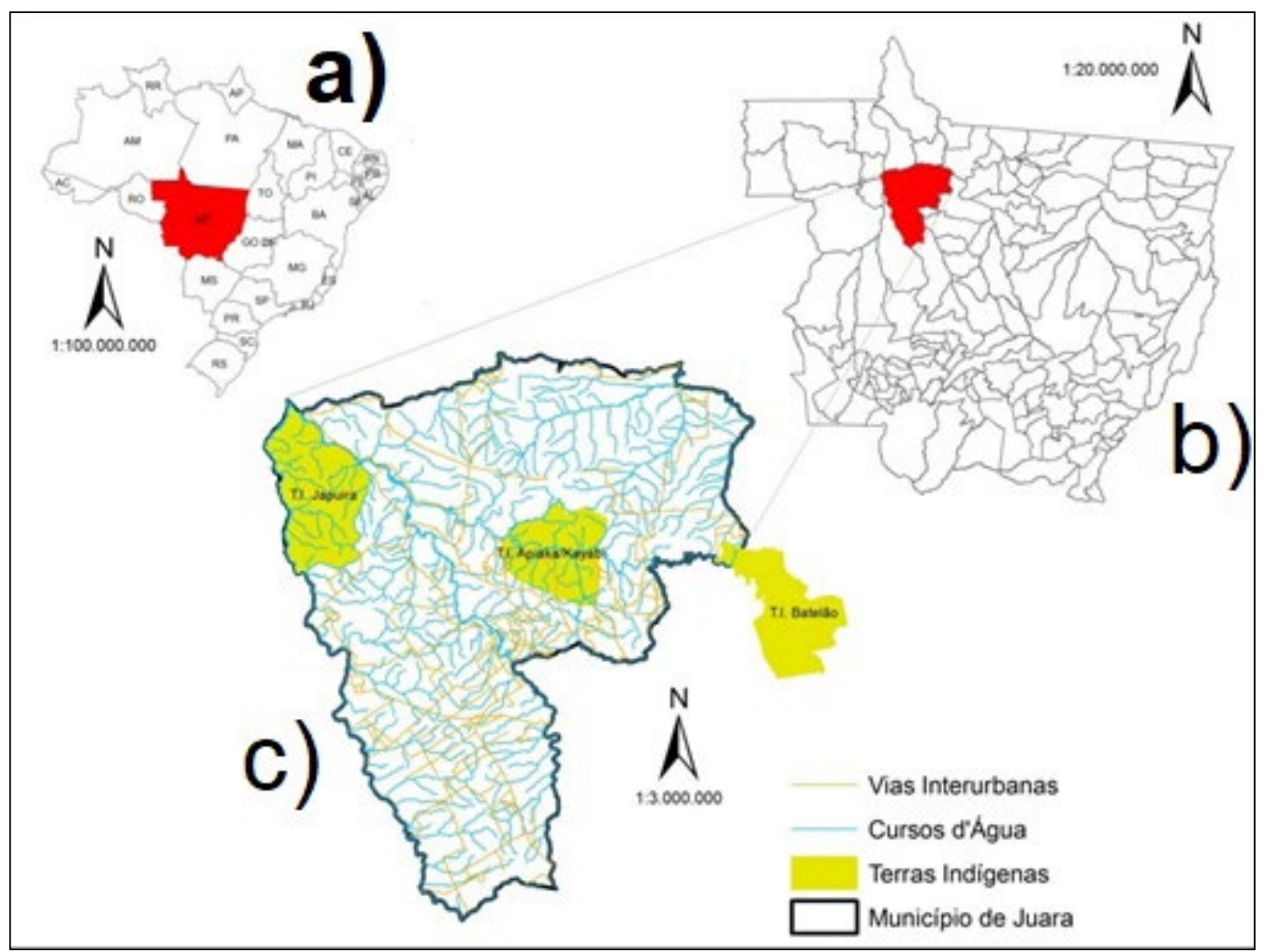

Fonte: Elaborado pelo autor com base em IBGE, 2016.

\subsection{Coleta de dados}

Para realizar o mapeamento dos focos de calor foi utilizado o banco de dados do Programa Queimadas do Instituto Nacional de Pesquisas Espaciais (INPE) com ênfase no monitoramentos dos Satélites Aqua - M - T, METOP - 8, NOAA - 18, NOAA - 19, NPP, NPP M - 375, TERRA - M - M, com propósito 
de identificar mensalmente os focos de calor durante o período de 01/01/2016 a 31/12/2017, totalizando dois (02) anos.

Os dados coletados foram exportados em formato shapefile para o Software ArcGis, onde recebeu tratamento acompanhado de técnico especializado.

A distribuição espacial dos focos de calor foi analisada no limite municipal do município de Juara - Mato Grosso, obtido no site do Instituto Brasileiro de Geografia e Estatística (IBGE). No programa ArcGis os dados foram inseridos e quantificados mensalmente e anualmente, transformados em 01 mapa. É possível então comparar as incidências em cada ano e mês.

\section{RESULTADOS}

No ano de 2016, representado pelo Gráfico 01, o mês de janeiro (28 focos) teve a menor incidência de focos de calor do ano. O período de abril a novembro teve incidência constante de fogo. Os meses de junho (400 focos), julho (1.217 focos), agosto (1.718 focos), setembro ( 2.368 focos) e outubro (740 focos) são os de maiores indicadores de queimadas.

Gráfico 01 - Incidência mensal de focos de calor no ano de 2016

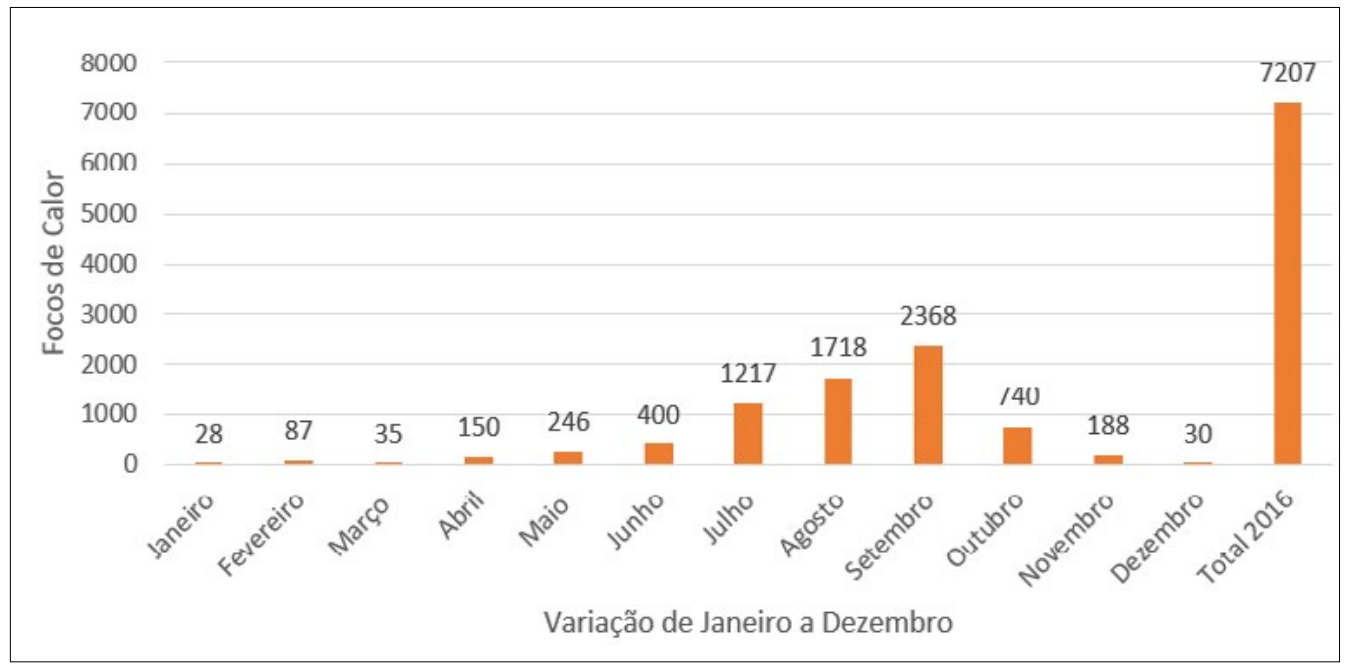

Fonte: Adaptado pelo autor com base em INPE Queimadas (2016 e 2017).

O ano de 2017 (Gráfico 02) apresenta 9.150 focos de calor com destaque aos meses de junho (407 focos de calor), julho (285 focos), agosto (2.255 focos), setembro (4.028 focos) e outubro (1.539 focos). 
Gráfico 02 - Incidência mensal de focos de calor no ano de 2017

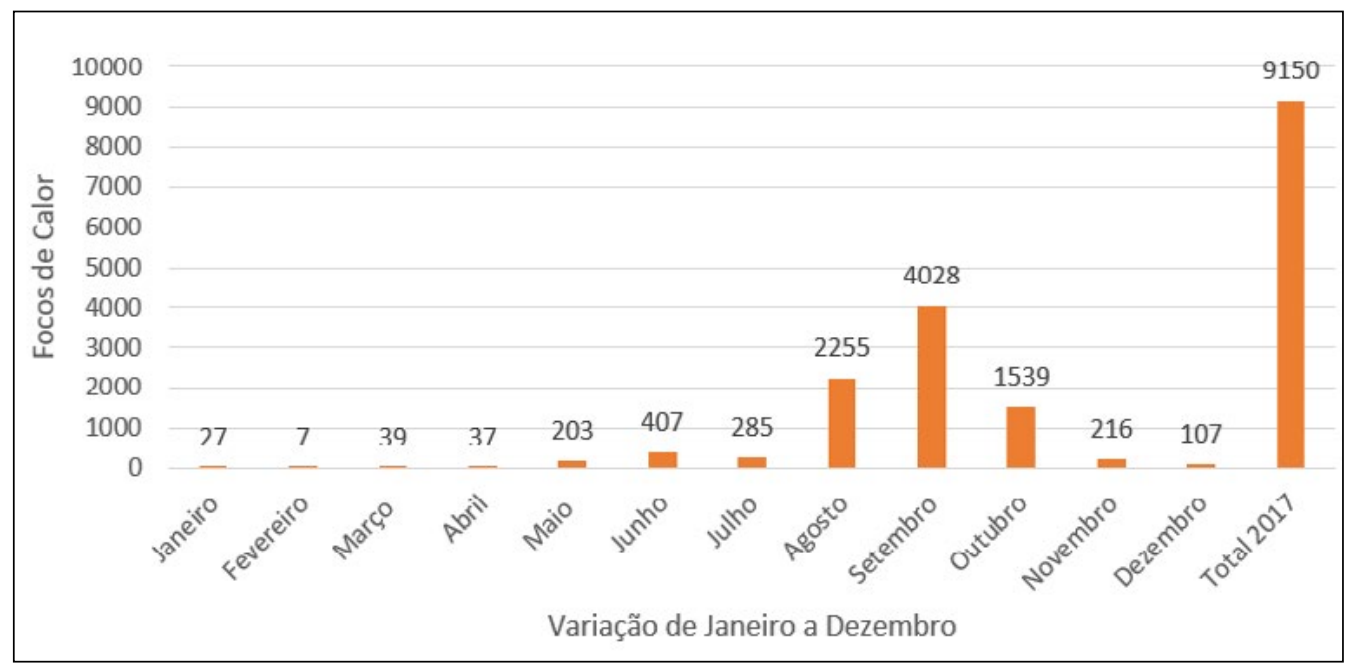

Fonte: Adaptado pelo autor com base em INPE Queimadas (2016 e 2017). 
Figura 2 - Mapa de focos de calor nos anos de 2016 e 2017 distribuídos pelo município de Juara - Mato Grosso

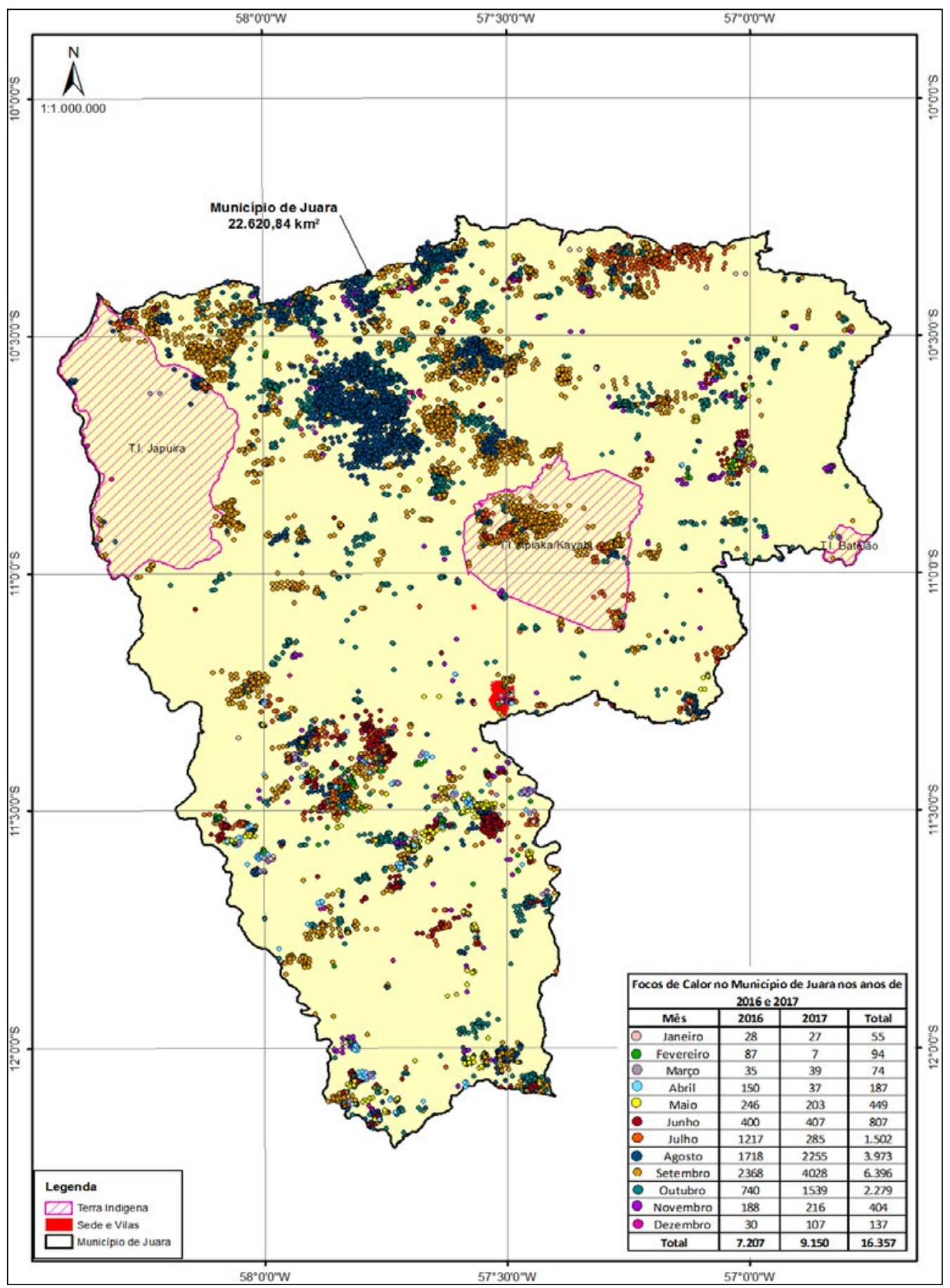

Fonte: IBGE (2007); INPE (2016 a 2017); Elaborado pelo autor. 


\section{DISCUSSÕES}

O ano de 2017 teve aumento de 21,24\% na incidência de focos de calor em relação ao ano de 2016. Vale destacar que os meses de agosto, setembro e outubro registram a maior incidência de focos de calor e que existem registros de focos de calor em todos os meses dos anos pesquisados.

De acordo com os dados coletados foi possível quantificar durante o período de 01/01/2016 a 31/12/2017 (02 anos) o total 16.357 focos de calor distribuídos sobre o município de Juara, Mato Grosso (Amazônia Legal).

O município de Juara está localizado na Amazônia Brasileira, onde o fogo tem sido aplicado como ferramenta de manejo para abertura de áreas agricultáveis, ampliação de áreas de pastagens e também para a manutenção de áreas já desmatadas (BOWMAN et al., 2008; COCHRANE et al., 1999; GIGLIO et al. 2006; KODANDAPANI et al., 2004; SORRENSEN, 2008; SILVA, 2018). A presença do fogo nesse ambiente pode ser problematizada pela prática de subsistência das atividades rurais, utilizado como ferramenta de manejo das áreas (SORRENSEN, 2008).

No Brasil atribui-se que a cultura do uso do fogo como técnica de manejo foi passada dos indígenas aos sertanejos, tanto no Cerrado como nos Campos Sulinos, biomas onde a queima é realizada para manejo de áreas de produção rural (FIDELIS; PIVELLO, 2011).

Os incêndios estão relacionados à invasão de fronteiras agrícolas e também a extração de madeira primária (TASKER; ARIMA; 2016), criação de gado bovino por pequenos produtores e pecuária extensiva por grandes fazendeiros (HOMMA et. al., 1998).

Por outro lado, a ação antropogênica é grande ameaça a conservação da floresta, que repercute a nível global ao regime climático da terra (SORRENSEN, 2008), contribuindo para que as florestas nativas possam ser esgotadas de forma plausível (XAUD; MARTINS; SANTOS, 2013).

\section{CONCLUSÕES}

A partir da análise e discussão dos dados coletados é possível concluir que:

1 - A pesquisa compreendeu o período de 01/01/2016 a 31/12/2017. Nesse ciclo de 02 anos foram quantificados 16.357 focos de calor distribuídos pelo município de Juara na Amazônia Legal.

2 - O ano de 2016 registrou 7.207 focos de calor. O ano de 2017 registrou aumento de $21,24 \%$ em relação a 2016 com 9.150 focos de calor.

3 - Todos os meses dos anos de 2016 e 2017 registraram focos de calor, sendo que os meses de julho, agosto, setembro e outubro registraram a maior quantidade de focos de calor. 
5 - Estudos de Fernandes et al., (2011) descrevem que o clima úmido e alta precipitação da Amazônia Legal não favorece incêndios naturais. Já as alterações climáticas e a ação humana influenciam a ocorrência de fogo em curtos espaços de tempo (ARAGÃO; SHIMABUKURO, 2010, COCHRANE; BARBER, 2009, LEWIS et al., 2011; SILVA et al., 2018).

5 - Há indícios de que o fogo no município de Juara tem sido utilizado como ferramenta do homem para manejo de áreas agricultáveis, ampliação de pastagens e também manutenção de áreas desmatadas. Estudos de Bowman et al., (2008), Cochrane et al., (1999), Giglio et al., (2006), Kodanpani et al., (2004), Sorrensen et al., (2008) comprovam que nas últimas décadas o fogo é empregado como técnica de manejo na Amazônia Brasileira para a manutenção de áreas de produção rural.

6-A base de dados elaboradas em formato shapefile pode ser utilizadas para estabelecer correlação com focos de calor por uso e ocupação do solo, temperatura, altitude, temperatura, tipos de cobertura vegetal, bioma, além de outras variáveis.

\section{REFERÊNCIAS}

ALVARADO, S.T. et al. Driver of occurrence in a mountains Brazilian Cerrado Savanna: Tracking long-term fire regimes using remote sensing. Ecological Indicators. V.78, P. 270-281, 2017.

ARAGÃO, L. O. C; SHIMABUKURO. The incidence of fire in Amazonian forests with implications for Redd. Science. V. 328, P. 1275-1278, 2010.

ARGAÑARAZ, J. P. et al. Human and biophysical drivers off ires in Semiarid Chaco mountains of Central Argentina. Science of The Environment. V.520, P. 1-12, 2015.

ARIMA, E.; VERÍSSIMO, A.; Brasil em Ação: ameaças e oportunidades econômicas na fronteira Amazônica. Imazon. 2002.

AVITABILE, S. C. et al. Systematic fire mapping is critical for fire ecology, planning and management: A case study in the semi-arid Murray Mallee, south-eastern Australia. Landscape and Urban Planning. V.117, p.81-91, 2013.

BOND, W. J.; KEELEY, J.E. Fire as a global "herbivore": The ecology and evolution of flammable ecosystems. Trends in Ecology \& Evolution. V. 20, 387-394, 2005. BOWMAN, M.S.; AMACHER, G. S.; MERRY, F. D. Fire use and prevention by traditional households in the Brazilian Amazon. Ecological Economics. V. 67, P. 117130, 2008.

BURROWS, N. D. Linking fire ecology and fire management in south-west Australian forest landscapes. Forest Ecology and Management. V. 255, p. 2394-2406, 2008. 
CHEN, D. et al. Mapping fire regimes in China using MODIS Active fire and Burned Area Data. Applied Geography. V.85, p. 14-26, 2017.

CHEMIN, B.F. Manual da Univates para trabalhos acadêmicos. $2^{\text {a }}$ Edição, Ed. Univates, Lajeado - RS, 2012.

COCHRANE, M. A. et al. Positive feedbacks in the fire dynamic of closed canopy tropical forests. Science. V. 284, P. 1832-1835, 1999.

COCHRANE, M. A.; BARBER, C. P. Climate change, human land use and future fires in the Amazon. Global Change Biology. V. 15, P.601-612, 2009.

COUTINHO, A.C. Dinâmica das queimadas no estado de Mato Grosso e suas relações com as atividades antrópicas e a economia local. Tese de Doutorado - USP. São Paulo, 2005.

FIDELIS; PIVELLO. Deve-se Usar o Fogo como Instrumento de Manejo no Cerrado e campos Sulinos. Disponível em: <http:/ / www.icmbio.gov.br/revistaeletronica/ index.php/BioBR/article/view/102>. Acesso em: 23 fev. 2017.

GIGLIO, L. et al. Global estimation of burned area using Modis active fire observations. Atmospheric Chemistry na Physics. V. 6, P.957-974, 2006.

HOMMA, A. K. O. et al. Redução dos desmatamentos na Amazônia: política agrícola ou ambiental? In: HOMMA, A.K.O (ed.). Amazônia: meio ambiente e desenvolvimento agrícola. Brasília, EMBRAPA-SPI, p.120-141, 1998.

IBGE - INSTITUTO BRASILEIRO DE GEOGRAFIA E ESTATÍSTICA. Mapas de Biomas e de Vegetação. Rio de Janeiro, 2004. Disponível em: <https:/ / ww2.ibge.gov. br/home/presidencia/noticias/21052004biomashtml.shtm.> Acesso em: 10 jul. 2017.

IBGE - INSTITUTO BRASILEIRO DE GEOGRAFIA E ESTATÍSTICA. Biomas Brasileiros. Rio de Janeiro, 2007. Disponível em: http:/ /7a12.ibge.gov.br/vamosconhecer-o-brasil/nosso-territorio/biomas.html. Acesso em: 10 jun. 2017.

IBGE - INSTITUTO BRASILEIRO DE GEOGRAFIA E ESTATÍSTICA. Queimadas Monitoramento e focos. Rio de Janeiro, 2016. Disponível em: https:/ / sidra.ibge.gov. br/tabela/894\#resultado. Acesso em: 10 jul. 2017.

INPE - INSTITUTO NACIONAL DE PESQUISAS ESPACIAIS. Bacos de dados de Queimadas (2016 a 2017). Disponível em: < http:/ / www.inpe.br/queimadas / bdqueimadas> Acesso em: 10 jan. 2018.

INDEA - INSTITUTO DE DEFESA AGROPECUÁRIA DO ESTADO DE MATO GROSSO, 2017. Relatório de Bovinos existentes no estado de Mato Grosso. Disponível em: <http://www3.indea.mt.gov.br/defesa-sanitaria-animal/downloads/ relatorio-de-bovinos-existentes/163417>. Acesso em: 23 jan. 2018. 
IMEA - INSTITUTO MATO GROSSENSE DE ECONOMIA AGRÍCOLA, 2017. Mapa das macrorregiões do Imea. Disponível em: <http:/ / www.imea.com.br/upload/ publicacoes/arquivos/justificativamapa.pdf $>$. Acesso em: 23 jan. 2018.

KODANDAPANI, N.; COCHRANE, M. A.; SUKUMAR, R. Conservation threat of increasing fire frequencies in the Western Ghats, India. Conservation biology, V.18, P. 1553-1561, 2004.

LARA, D. B. et al. Avaliação de Fragmentos de lenhos carbonizados de Araucariaceae por meio de Termogravimétrica e Infravermelho associadas à Análise Multivariada.

Revista Química Nova. Vol. 40, N8, p. 895-901, 2017.

LEWIS, S. L. et al. The 2010 amazon drought. Science. V. 331, P. 554-554, 2011.

MATO GROSSO. Relatório Técnico de monitoramento de focos de calor de $\mathbf{2 0 1 5}$ No03 do Estado de Mato Grosso. Secretaria de estado de Meio Ambiente. Sema, Mato Grosso, Brasil, 2015.

MORENO, G.; Políticas e estratégias de ocupação. In: MORENO, G.; HIGA, C. S. (Orgs.). Geografia de Mato Grosso. Cuiabá. P. 52-71, 2005.

PAUSAS, J. G. Evolutionary fire ecology: lessons learned from pines. Trend in Plant Science. V.20, p. 318-324, 2015.

PYNE, S. J; Vestal Fire: An Environmental History. Told trough Fire, of Europe And Europe's Encounter with the world. University of Washington press. 2012.

SALGADO-LABOURIAU, M. L.; FERRAZ-VINCENTINI, K. R.; Fire in the Cerrado 32,000 years ago. Current Research in the Pleistocene, 11: 85-87. 1994.

SCOTT, A.C. et al. Fire on Earth: An Introduction. 1a Edition, Wiley Blackwell, 2014.

SILVA, T. B.; ROCHA, W. J. S; ANGELO, M. F. Quantificação e análise espacial dos focos de calor do Parque Nacional da Chapada Diamantina - BA. Anais do XVI Simpósio Brasileiro de Sensoriamento Remoto do INPE, Foz do Iguaçu, PR, Brasil, 2013.

SILVA, S. S. et al. Dynamics of forest fires in the southwestern Amazon. Forest Ecology and Management. V.424, P. 312-322, 2018.

SORRENSEN, L. C. Contributions of fire use study to land use/cover change frameworks: understanding landscape change in agricultural frontiers. Human Ecology, V. 32, P. 395-420, 2004.

SORRENSEN, L. C. Potential hazards of land policy: Conservation, rural development and fire use in the Brazilian Amazon. Land Use Policy. V. 26, P. 782-791, 2009.

TASKER, K. A.; ARIMA, E. Fire regimes in Amazonia: The relative roles of policy and precipition. Anthropocene. V. 14, P. 46-57, 2016. 
XAUD, H. A. M; MARTINS, F. S. R.; SANTOS, J. R. Tropical forest degradation by mega-fires in the northern Brazilian Amazon. Forest Ecology Management. V. 294, P. 97-106, 2013.

ZYLBERSZTAJN, D. Análise de sistemas do agronegócio: Origem, Evolução e Perspectivas de pesquisa. Revista de Administração da Universidade de São Paulo. V.52, Edição 1, P. 114-117, 2017. 